\title{
Nous y voilà, la CPS FMH arrive
}

Dès juillet 2009, les premières cartes de professionnel de santé de la FMH, ou CPS FMH, seront prêtes. Cette nouvelle carte de médecin et de membre FMH sera livrée dans la version de base «carte de médecin personnelle avec certificat numérique avancé». Les membres de la FMH recevront la CPS FMH gratuitement!

Une série d'articles sera consacrée à la CPS $\mathrm{FMH}$, à ses fonctionnalités et possibilités d'utilisation. Les articles seront dans le même temps accessibles sur www.fmh.ch, ce qui vous permettra de vous informer à tout moment. A partir de juillet 2009, une aide téléphonique et par e-mail sera aussi proposée pour toutes les questions liées à la mise en service et à l'utilisation de la carte.

\section{Arzt/Ärztin · Médecin · Medico · Physician}

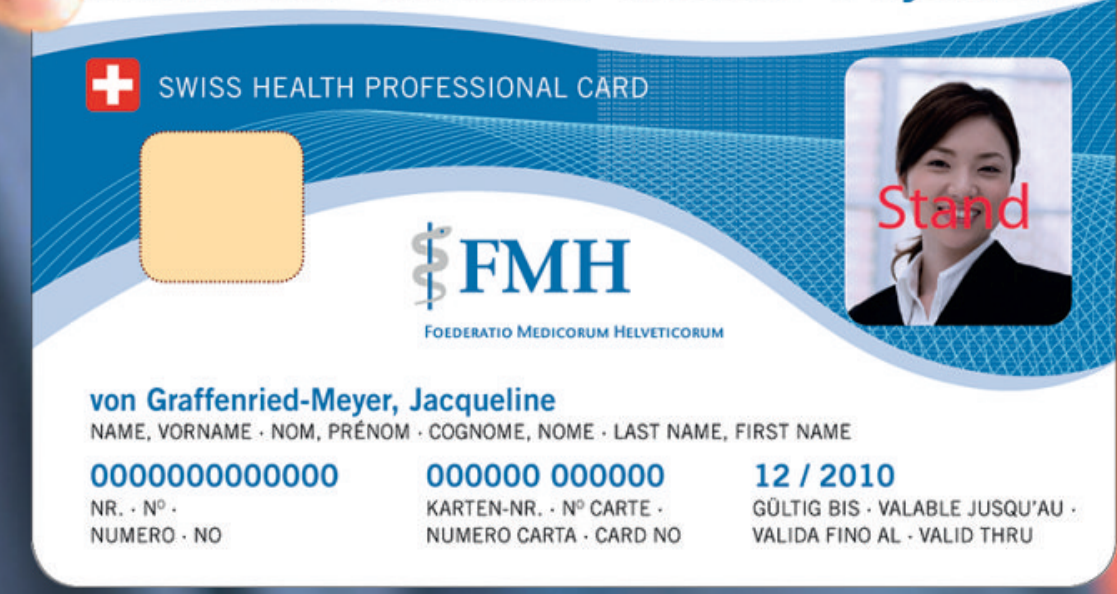

\section{La CPS FMH - une carte avec trois fonctionnalités}

Dans le format d'une carte de crédit et avec un design plaisant, la CPS FMH fait son arrivée. Elle n'est pas seulement la nouvelle carte de membre de la Fédération des médecins suisses, elle offre en effet plusieurs autres utilités. La CPS FMH est:

- la première carte suisse de professionnel de santé pour tous les médecins qui exercent en Suisse

Grâce à un processeur crypto-chip conforme aux exigences de la loi suisse sur la signature électronique (SCSE), la carte contient les certificats prévus (certificat avancé, certificat qualifié et aussi ultérieurement le certificat $\mathrm{CVC}$ ). Le certificat avancé permet entre autres de signer les courriels et les documents et d'envoyer les courriels de façon cryptée. Le certificat qualifié permet en outre d'apposer sa signature de façon juridiquement valable sur les documents et les courriels. Enfin, le certificat CVC devrait aussi donner l'accès à la carte d'assuré dont la distribution à la population suisse est prévue à partir de 2010.

- la nouvelle carte personnelle de membre et de médecin pour les membres de la FMH

La CPS remplace l'ancienne carte jaune de médecin. Comme la CPS FMH contient désormais une photo numérisée, il n'est plus nécessaire de présenter une autre pièce d'identité, par exemple au moment d'acheter des médicaments dans une pharmacie à l'étranger.

- la carte de médecin personnelle pour les médecins non affiliés à la FMH

Les médecins non affiliés à la FMH peuvent également recevoir une carte de médecin personnelle. Celle-ci correspond pour l'essen- tiel à la carte de membre, à deux différences près: La carte n'est pas munie du logo FMH et elle est payante.

\section{Comment recevoir sa CPS FMH}

La FMH enverra les formulaires de demande de la CPS FMH à tous ses membres. Faites-nous parvenir le formulaire «DEMANDE d'une carte CPS FMH» muni de votre signature et d'une photo d'identité récente et vous serez bientôt en possession de votre CPS FMH! Pour des raisons logistiques, l'envoi des formulaires aux membres FMH se fait de manière échelonnée:

- Début mai, tous les membres de la FMH qui sont actifs dans un organe de la FMH (membres du Comité central et de la Commission de gestion, délégués et délégués suppléants de la Chambre médicale et de l'Assemblée des délégués) recevront leurs formulaires de demande.

- Les membres de la FMH qui se rendront à l'assemblée annuelle de la Société Suisse de Médecine Interne à Bâle entre le 13 et le 15 mai 2009 pourront directement se procurer leurs formulaires au stand de la FMH, niveau 2, juste en face du stand de la SSMI. Le 13 mai, il vous sera même possible, à l'occasion de deux workshops organisés en français et en allemand, de vous informer sur la CPS FMH et ses fonctionnalités, ainsi que de l'expérimenter en direct. Si vous n'avez pas de photo d'identité, il sera possible sans problème d'en faire à un prix réduit, juste à côté du Centre des congrès. Renseignez-vous au stand FMH pour obtenir des informations plus précises sur l'offre FMH «photo d'identité».

- Les autres membres de la FMH recevront leurs formulaires de demande de la CPS FMH mi-août, mi-septembre ou mi-octobre. Les médecins FMH en pratique indépendante seront prioritaires. 


\section{Vos expériences nous intéressent}

Moins vous mettrez de temps à renvoyer votre demande, plus vous recevrez votre CPS FMH dans des délais rapides. Pour des raisons techniques de fabrication, les premières cartes seront livrées courant juillet. Passé ce délai, leur livraison interviendra dans l'intervalle de quatre semaines après réception de la demande. Vos expériences pratiques avec la CPS FMH nous intéressent et c'est pourquoi nous invitons les premiers bénéficiaires de la carte à participer à un essai pilote. Cette démarche ne vous prendra que peu de temps, mais pourra vous être utile: votre feedback nous permettra d'améliorer les fonctionnalités et les prestations de services dans le cadre de la nouvelle carte de médecin et de membre de la FMH. Vous obtiendrez des informations plus détaillées sur l'essai pilote conjointement avec votre CPS FMH.

Erika Flückiger,

Responsable des Services de la FMH

\section{La CPS FMH et ses spécificités vous seront présentés dans le prochain article.}

\section{Nécrologie}

\section{In memoriam}

Prof. Philip Poole-Wilson stirbt unerwartet

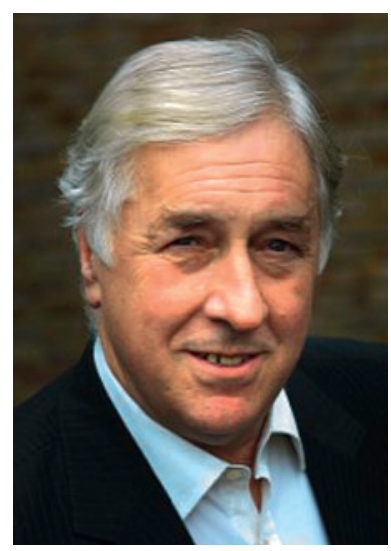

Prof. Poole-Wilson, weltbekannter Kardiologe und ehemaliger Präsident der European Society of Cardiology, ist am 4. März 2009 auf dem Weg zu seiner Arbeit in London unerwartet zusammengebrochen und kurz darauf verstorben.

Philip Poole-Wilson war Vorsteher des Departements für Herzmedizin am National Heart and Lung Institute des Imperial College in London und Honorary Consultant Physician des Royal Brompton \& Harefield Hospital und Simon Marks Professor of Cardiology der British Heart Foundation.

Prof. Poole-Wilson war ein weltweit anerkannter Experte für Herzinsuffizienz und ko- ronare Herzkrankheit und hat die moderne Kardiologie durch seine eigenen Forschungsbeiträge, Diskussionen und seine klare Haltung mitgeprägt. Er war bekannt für seine sichere und kritische Einschätzung neuer Entwicklungen und ein gefürchteter Debattierer an internationalen Kongressen.

Philip Poole-Wilson hat auch in vielen medizinischen Gesellschaften eine wesentliche Rolle gespielt, so als Präsident der World Heart Federation von 2003 bis 2005 und der European Society of Cardiology von 1994 bis 1996. Er war als Founding Chair der British Society for Heart Failure und in zahlreichen Editorial Boards verschiedenster internationaler und britischer Zeitschriften tätig, so im «Journal of the American College of Cardiology» u.a.m. Prof. Poole-Wilson hat mit seiner Expertise zu vielen exzellenten Lehrbüchern beigetragen und wird im PubMed mit mehr als 500 Publikationen gelistet.

Philip Poole-Wilson hat verschiedene Preise während seiner Karriere gewonnen, so die McKinsey Medal der British Cardiovascular Society im Jahre 2007, den Prix Européen de Médicine des Institut de Sciences et de la Santé in Paris im Jahr 2001.

Philip Poole-Wilson ist völlig unerwartet verschieden. Noch Mitte Februar war er ein prominentes Faculty Member am Cardiology Update in Davos, wo er drei Vorträge gehalten und sehr lebhaft an den Diskussionen teilgenommen hat.

Thomas F. Lüscher, Zürich 